Review Article

\title{
Synaptojanin 1 Mutation in Parkinson's Disease Brings Further Insight into the Neuropathological Mechanisms
}

\author{
Valérie Drouet ${ }^{1,2}$ and Suzanne Lesage ${ }^{1}$ \\ ${ }^{1}$ Sorbonne Universités, UPMC (Paris 6), UMR S 1127, Inserm U 1127, CNRS UMR 7225, and ICM, 75013 Paris, France \\ ${ }^{2}$ Hôpital Pitié-Salpêtrière, Institut du Cerveau et de la Moelle Epinière ICM, 4ème Étage, 47 Boulevard de l'Hôpital, 75651 Paris, France
}

Correspondence should be addressed to Valérie Drouet; v.drouet-ihu@icm-institute.org

Received 9 July 2014; Accepted 26 August 2014; Published 16 September 2014

Academic Editor: Hiroyuki Tomiyama

Copyright (C) 2014 V. Drouet and S. Lesage. This is an open access article distributed under the Creative Commons Attribution License, which permits unrestricted use, distribution, and reproduction in any medium, provided the original work is properly cited.

\begin{abstract}
Synaptojanin 1 (SYNJ1) is a phosphoinositide phosphatase highly expressed in nerve terminals. Its two phosphatase domains dephosphorylate phosphoinositides present in membranes, while its proline-rich domain directs protein-protein interactions with synaptic components, leading to efficient recycling of synaptic vesicles in neurons. Triplication of SYNJ1 in Down's syndrome is responsible for higher level of phosphoinositides, enlarged endosomes, and learning deficits. SYNJ1 downregulation in Alzheimer's disease models is protective towards amyloid-beta peptide $(\mathrm{A} \beta)$ toxicity. One missense mutation in one of SYNJ1 functional domains was recently incriminated in an autosomal recessive form of early-onset Parkinson's disease (PD). In the third decade of life, these patients develop progressive Parkinsonism with bradykinesia, dystonia, and variable atypical symptoms such as cognitive decline, seizures, and eyelid apraxia. The identification of this new gene, together with the fact that most of the known PD proteins play a role in synaptic vesicle recycling and lipid metabolism, points out that synaptic maintenance is a key player in PD pathological mechanisms. Studying PD genes as a network regulating synaptic activity could bring insight into understanding the neuropathological processes of PD and help identify new genes at fault in this devastating disorder.
\end{abstract}

\section{Introduction}

Synaptojanin 1 (SYNJ1) was discovered in 1994 as a $145 \mathrm{kDa}$ protein that interacts with growth factor receptor-bound protein 2 (Grb2) and a phosphoprotein involved in synaptic vesicle endocytosis and recycling $[1,2]$. One year ago, a SYNJ1 mutation was incriminated for the first time in autosomal recessive early-onset Parkinson's disease (PD) with atypical symptoms $[3,4]$. During this time, numerous studies were conducted on SYNJ1 and helped us better understand the roles of this multifunctional protein. This review will discuss how SYNJ1 operates in pre- and postsynaptic compartments to modulate synaptic activity, as well as its implication in different neurological disorders. SYNJ1 involvement in $\mathrm{PD}$ is also examined within the network of other known PD proteins.

\section{SYNJ1 Gene and Protein Organization}

The SYNJ1 gene is located on chromosome 21q22.11 [5] and spans $99.29 \mathrm{~kb}$ of genomic DNA. Two isoforms of 170 and $145 \mathrm{kDa}$ have been widely studied $[2,6,7]$ (isoform a: NP_003886.3, 1612 amino acids; and isoform b: NP_982271.2, 1350 amino acids). They are generated from two open reading frames (ORFs) separated by an in-frame TAA stop codon [8]. They are both ubiquitously expressed, but the $145 \mathrm{kDa}$ isoform is found in very high concentrations in brain [7, 8 ] where it is localized on coated endocytic intermediates in nerve terminals $[2,9]$. Both isoforms harbor multiple functional domains: a suppressor of actinl Sacl-like domain on their N-terminal, a $5^{\prime}$-phosphatase domain in the center, and a C-terminal proline-rich domain (PRD). The longer $170 \mathrm{kDa}$ isoform contains an additional PRD translated from 
the second ORF (Figure 1). There are two additional SYNJ1 isoforms listed in RefSeq (isoform c: NP_001153774.1, 1295 amino acids; isoform d: NP_001153778.1, 1526 amino acids) that are of unknown functional relevance. Despite isoforms c and $\mathrm{d}$ have a shorter $\mathrm{N}$-terminus and a distinct $\mathrm{C}$-terminus and are shorter than isoform a, they contain the same functional domains as isoforms $b$ and a, respectively.

2.1. Inositol Phosphatase Functions. Inositol lipids are essential components of eukaryotic membranes and important intracellular second messengers that can be regulated by phosphorylation. Inositol phosphatases remove phosphate groups from phosphoinositides (e.g., phosphorylated inositol lipids) and play important roles in lipid signaling, cell signaling, and membrane trafficking [10]. SYNJ1 contains two consecutive inositol phosphatase domains, the Sacl and the $5^{\prime}$-phosphatase domains (Figure 1). The N-terminal Sacl domain, homologous to the yeast SacIp, dephosphorylates predominantly phosphatidylinositol monophosphates present in cell membranes, including those of the Golgi apparatus and endosomes, to recruit proteins. The central $5^{\prime}-$ phosphatase domain dephosphorylates phosphatidylinositol bis- or trisphosphates, localized in plasma membranes, to activate downstream pathways $[6,8,11,12]$. In neurons, SYNJ1 dual phosphatase activity is required for efficient synaptic vesicle endocytosis and reavailability at nerve terminals [13].

2.2. Protein-Protein Interactions. Many intracellular proteins contain proline-rich sequences that serve as binding sites for Src homology 3 (SH3) domains. Based on its $\mathrm{SH} 3$ binding ability, SYNJ1 was initially identified as interacting with Grb2 [1]. In fact, SYNJ1 contains a 250 amino acid PRD at its Cterminus, with at least five potential SH3 domain-binding consensus sequences $[8,14]$. The $170 \mathrm{kDa}$ isoform harbors an additional smaller PRD with at least three additional SH3 binding sites [7] (Figure 1).

Besides Grb2, the C-terminal region common to both SYNJ1 isoforms interacts with the $\mathrm{SH} 3$ domains of a variety of proteins implicated in endocytosis, subcellular targeting, and signaling: endophilin, amphiphysin, syndapin/pacsin, intersectin, and many others [15-20]. Through a SH3-PRD interaction, endophilin recruits SYNJ1 to endocytic sites to promote dephosphorylation of phosphatidylinositol 4,5bisphosphates by way of SYNJ1 $5^{\prime}$-phosphatase activity [21] The $170 \mathrm{kDa}$ splice variant bears an additional C-terminal tail that contains binding sites for clathrin, clathrin adaptor protein complex 2 (AP2) via three types of binding motifs (WxxF, FxDxF, and $\mathrm{DxF}$ ), and the epidermal growth factor receptor pathway substrate 15 (Eps15) through asparagineproline-phenylalanine (NPF) domain $[9,22,23]$ (Figure 1). The complex AP2 is a protein interaction hub binding to all the endocytic components, including Eps15, necessary for clathrin-mediated endocytosis [22].

\section{SYNJ1 Multiple Functions}

Because of its different functional domains, SYNJ1 plays a key role in nerve terminals, coupling endocytic vesicle fission, and phosphoinositide dephosphorylation, but it has also been shown that SYNJ1 takes part in similar mechanisms in cone photoreceptors [24-27], hair cells [28], podocyte foot processes [29], and, more recently and unexpectedly, T cells [30].

3.1. Functions in Neurons. SYNJ1 functions in neurons are mainly promoted by the $145 \mathrm{kDa}$ isoform, since the $170 \mathrm{kDa}$ isoform is undetectable in the adult rat brain [7]. Most of the studies focused on synapses, since SYNJ1 $145 \mathrm{kDa}$ is highly enriched in presynaptic nerve terminals and, like dynamin, interacts with amphiphysin and undergoes dephosphorylation after nerve terminal depolarization $[2,8]$. It also interacts with endophilin and, together, they are rapidly recruited to clathrin-coated pits during prolonged stimulation $[6,13]$.

SYNJ1-deficient mice exhibit neurological defects such as severe weakness, ataxia, spontaneous epileptic seizures, and poor motor coordination and die shortly after birth [11]. Likewise, mutations in unc-26, the single synaptojanin gene in C. elegans, give rise to small animals which are moving backwards with a jerky motion and frequently coil and have reduced numbers of enteric muscle contractions [31]. Studies of these mutants, lamprey giant reticulospinal axons microinjected with antibodies against synaptojanin [32], and yeast inactivated for synaptojanin-like proteins [33] have revealed increased levels of phosphatidylinositol 4,5bisphosphates, an increased number of clathrin-coated vesicles, and a hypertrophy of the actin-rich matrix at endocytic zones. This shows that, in the brain, regulation of phosphoinositide levels by the SYNJ1 $5^{\prime}$-phosphatase domain is essential for proper vesicle trafficking and coating/uncoating of endocytic vesicles [11,34]. Through dephosphorylation of phosphatidylinositol 3- and 4-monophosphate [12, 34], the SYNJ1 Sacl domain participates in actin cytoskeleton polymerization/depolymerization and is mostly required during brief neuronal stimulation [13]. To a lower extent, Sacl activity is also an arbiter of phosphatidylinositol 3,5-bisphosphates levels, playing an important role in early and late endosomes turnover [35]. In addition, another role has been identified for SYNJ1 postsynaptically: it is involved in the internalization of AMPA receptors in postsynaptic neurons [36].

Therefore, SYNJ1 not only is involved in endocytic and postendocytic mechanisms presynaptically but is also participating in the signal transmission through postsynaptic reorganization.

3.2. The Particular Case of Sensory Neurons. In the particular case of photoreceptor and hair cells, sensory information transmission relies on ribbon synapses. These "unconventional" synapses have very high rates of continuous exocytosis and therefore need to have efficient endocytosis and vesicle recycling mechanisms [37].

Mutation in SYNJ1 in a Zebrafish vision mutant $(n r c)$ showed unanchored ribbons and reduced numbers and abnormal distribution of synaptic vesicles that are scattered within a dense cytoskeletal matrix in cone photoreceptors [25]. Additional studies in Zebrafish confirmed that SYNJ1 is required for proper membrane and protein trafficking at 


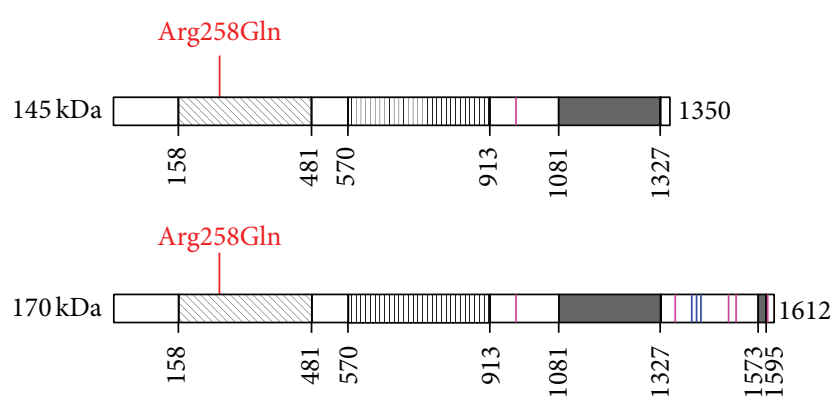

Functional domains:

Interaction domains:

$$
\begin{aligned}
& \text { Sac1 } \\
& 5^{\prime} \text {-Phosphatase }
\end{aligned}
$$

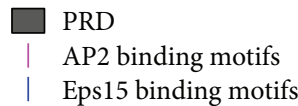

FIGURE 1: Functional and interaction domains of the two major isoforms of SYNJ1. The $145 \mathrm{kDa}$ (top) and the $170 \mathrm{kDa}$ (bottom) SYNJ1 isoforms harbor two functional inositol phosphatase domains, an Nterminal Sacl domain and a more central $5^{\prime}$-phosphatase domain. Several protein-protein interaction domains are found in the Cterminal part of the proteins: one or two PRD domains, AP2 binding motifs (WxxF, FxDxF, and DxF, in pink), and Eps15 binding motifs (NPF: asparagine-proline-phenylalanine, in blue). The homozygous mutation Arg258Gln, found in Parkinson's disease patients, is indicated in red. Numbers indicate the amino acid positions along the proteins. Sacl: suppressor of actin1; PRD: proline-rich domain; AP2: adaptor protein complex 2; Eps15: epidermal growth factor receptor pathway substrate 15 .

the ribbon synapses in cones $[24,26]$. A mutation was also found in another Zebrafish with a vestibular deficit (comet) [28]. This model showed that SYNJ1 plays a critical role in facilitating vesicle recycling at ribbon synapses through controlling the number of vesicles released and timing of release. These roles discovered in Zebrafish have yet to be confirmed in mammalian models.

3.3. Functions in Other Cell Types. In the kidney, adjacent podocytes form an epithelial barrier via their foot processes, which are connected by a thin diaphragm (the slit diaphragm) for filtering plasma into the urinary space. In podocytes, only the $170 \mathrm{kDa}$ isoform of SYNJ1 is expressed, and, like in neurons, SYNJ1 participates in endocytosis with its interacting partners dynamin and endophilin by acting on phosphoinositides and actin filaments [29]. This is required for an efficient glomerular filtration and thus for proper renal function.

Recently, SYNJ1 has been reported as a potential regulator of allogeneic $\mathrm{T}$ cell responses [30]. This phenomenon can be triggered after transplantation from a genetically different person. The level of SYNJ1 mRNA was reduced after allogeneic stimulation of naïve $\mathrm{T}$ cells [30]. The authors believe that this reduced expression level is due to specific miRNA targeting the SYNJ1 transcript. Knockdown of SYNJ1 in allogeneically stimulated $\mathrm{T}$ cells confirmed its role in T cells proliferation and cytokine responses [30].

\section{SYNJ1 in Down's Syndrome (DS) and Alzheimer's Disease (AD)}

The critical importance of SYNJ1 at synapses has led multiple teams to investigate its role in neurological disorders such as $\mathrm{DS}$ and $\mathrm{AD}$. It became clear that a proper dosage of this gene was essential for good synaptic function.

4.1. SYNJ1 Trisomy in DS. DS, also known as trisomy 21, is the most common genetic cause of mental retardation and is caused by overexpression of one or several genes on chromosome 21. Along with the early development of $\mathrm{AD}$ pathology and muscle hypotonia, mental retardation occurs in all DS-affected individuals, whereas other phenotypes (e.g., congenital heart defects) occur in a fraction of patients [38]. Linkage analysis led to defining small chromosome 21 subregions as responsible for mental retardation, and SYNJ1 became a strong candidate gene [39]. Using a DS mouse model carrying a partial trisomy of mouse chromosome 16 (conserved with the long arm of human chromosome 21), it was shown that SYNJ1 was overexpressed in DS mouse brains, which was associated with higher levels of phosphatidylinositol 4,5-bisphosphates phosphatase activity and learning deficits [39]. Additional studies in human blood and Drosophila also confirmed the involvement of SYNJ1 in DS $[40,41]$. In particular, its triplication triggers abnormal synaptic morphology in fly neuromuscular junctions [40] and enlargement of early endosomes in lymphoblastoid cell lines derived from DS patients [41]. These endosomal abnormalities have been implicated in the early development of $\mathrm{AD}$ pathology in $\mathrm{DS}$ patients, but amyloid precursor protein (APP, also triplicated in DS) overexpression alone is not responsible for inducing endosomal enlargement in DS lymphoblastoid cells [41]. Measurement of SYNJ1 protein levels in DS-affected brains showed higher levels compared to matched controls, which is in agreement with SYNJ1 triplication in DS [42]. Moreover, in brains from individual with DS + AD pathology, levels of SYNJ1 are even higher and correlate with levels of amyloid-beta peptide $(\mathrm{A} \beta)$, whereas SYNJ1 levels are reduced in sporadic AD brains. The authors suggest that higher $\mathrm{A} \beta$ level could reduce SYNJ1 turnover in $\mathrm{DS}+\mathrm{AD}$ brains [42]. However, there are other genes that are triplicated in DS, and they could be involved, alone or together with SYNJ1, to explain the deficits observed in DS patients.

4.2. SYNJ1 in AD. DS patients, who carry triplication of both SYNJ1 and APP, develop early-onset AD [38]. This could be the result of overexpression of APP only, but some lines of evidence argue in favor of the combined effects of these two genes in the development of AD pathology. Both $\mathrm{A} \beta$ and SYNJ1 trigger internalization of AMPA receptor, which could provoke synaptic dysfunction $[36,43,44]$. In hippocampal cultures, addition of $\mathrm{A} \beta$ oligomers provoked a loss of dendritic AMPA receptors, via calcineurin-mediated endocytosis [44]. On the contrary, hippocampal neurons from SYNJ1 knockout mice showed more surface-exposed 
AMPA receptors [36]. Additionally, downregulation of phosphatidylinositol 4,5-bisphosphates enhances the production of $\mathrm{A} \beta 42$, while haploinsufficiency of SYNJ1 protects cells from the neurotoxic actions of $\mathrm{A} \beta 42$ [45]. The beneficial impact of SYNJ1 reduction in AD was confirmed in a mouse model of AD [46]. In these animals, hemizygous deletion of SYNJ1 rescued deficits in learning and memory. Moreover, genetic disruption of SYNJ1 attenuated $\mathrm{A} \beta$ oligomer-induced changes in dendritic spines of cultured hippocampal neurons [46]. This protective effect was shown as a result of a decrease in amyloid plaque burden mediated through accelerating endosomal/lysosomal degradation of $\mathrm{A} \beta$ [47]. These data underline the potential of SYNJ1 reduction as a possible therapeutic strategy to counteract $\mathrm{AD}$ pathology.

\section{SYNJ1 Mutation in Parkinson's Disease (PD)}

An abnormally high level of SYNJ1 is potentially responsible for several pathological features in DS, and reduction of this protein is being investigated as a therapeutic strategy to counteract $\mathrm{AD}$. But what happens when this protein is mutated? Several studies have linked bipolar disorder (BPD) to chromosomal region 21q22 containing SYNJ1 in a subset of families. Additionally, genes coding for proteins involved in the regulation of synaptic vesicle function are potential candidates for the development of psychiatric disorders. Therefore, SYNJ1 was found as a good candidate for BPD. Nevertheless, after screening about 230 patients with BPD, Lachman's team failed to statistically implicate SYNJ1 in BPD $[48,49]$. It was only last year that a mutation in SYNJ1 gene was associated, for the first time, to a neurodegenerative disorder, PD.

5.1. SYNJ1-Associated PD Mutation. In June 2013, using homozygosity mapping followed by exome sequencing, two teams independently identified the same homozygous mutation, Arg258Gln, in two consanguineous families, one Italian (from Sicilia) and one Iranian, suffering from autosomal recessive early-onset Parkinsonism [3, 4].

This missense Arg258Gln mutation that localizes in exon 5, within the Sacl domain of the protein (Figure 1), is predicted to be damaging by multiple prediction programs, and the arginine in position 258 is conserved in thirteen SYNJ1 orthologs and five Sacl-like domains containing proteins [3, 4]. Additionally, this mutation impairs the Sacl phosphatase activity targeting phosphatidylinositol monophosphate, suggesting that impaired synaptic vesicle recycling could be involved in PD pathology [3].

Screening of all exons in 138 additional patients, among which 46 presented with complex early-onset Parkinsonism, did not identify any other homozygous or compound heterozygous mutation in SYNJ1 [3, 4]. A team from Germany screened 792 PD patients (mostly Germans) and could not find any mutation in SYNJ1 exon 5 [50]. However, sequencing of the whole SYNJ1 coding sequence was missing. In addition, there were only 50 out of the 792 patients who had an age at onset $<30$ years, which was found to be an important feature in SYNJ1-associated PD cases (Table 1). Arg258Gln was also absent from 1268 control chromosomes (180 healthy controls from southern Italy [4], 96 controls from Iranian ancestry [3], 92 Caucasian neurologically normal individuals [3], and 266 controls from EPIPARK cohort $[50,51])$ and absent from multiple public databases representing more than 13,000 chromosomes $[3,4]$.

Recently, a third family was identified with the same homozygous Arg258Gln mutation in two siblings [52]. This family, from Naples in Italy, was not consanguineous, and haplotype study showed that the mutation had arisen independently in the ancestors of the two Italian families $[4,52]$.

SYNJ1 was named PARK20 (Online Mendelian Inheritance in Man, OMIM, 615530), even though mutations in this gene are extremely rare so far. To date, six early-onset PD patients (from three families with two affected siblings each) are carrying the homozygous Arg258Gln mutation. Their parents are all heterozygous for this variant while unaffected siblings are homozygous carriers for the wildtype allele or heterozygous mutation carriers [3, 4, 52]. Screening of all SYNJ1 coding regions in additional earlyonset PD is mandatory, and particular attention should be paid to potential copy number variations and mutations at the compound heterozygous state.

5.2. SYNJ1-Associated PD Phenotype. A phenotypic variability is observed in the three families presenting SYNJ1 mutation. Nevertheless, PARK20 families can be described as early-onset atypical Parkinsonism, with onset in the third decade of life, and severe progression in the first stages followed by stabilization in later stages [53]. Main clinical features combine tremor, dystonia, bradykinesia, and a poor response to levodopa treatment. Additional atypical signs such as seizures, cognitive impairment, developmental delay, and oculomotor disturbances are variable. Indeed, generalized seizures are seen in the Iranian siblings while only one of the Italian affected patients suffered of an episode of clonic seizures. Eyelid apraxia is seen in both Iranian and Sicilian families but is absent from the Neapolitan family. Important and mild cognitive decline are observed in the Sicilian and Neapolitan families, respectively, but not described in the Iranian siblings. Finally, only the Neapolitan siblings had mild delay in reaching the child developmental milestones $[3,4,52]$. Of importance, the six SYNJ1-mutated patients were examined at different stages of disease progression, did not always undergo the same tests, and were taking different treatments; it could account for some of the observed clinical differences.

The clinical features of these six patients are summarized in Table 1.

5.3. Synaptic Vesicle Recycling in PD. The functions of SYNJ1 in synaptic vesicle recycling and actin dynamics in pre- and postsynaptic compartments are of high interest to understand the physiopathology of PD and, to a larger extent, the role of lipid metabolism in neurological disorders. There is mounting evidence that synaptic vesicle trafficking pathways are implicated in PD mechanisms. Most of the proteins involved 


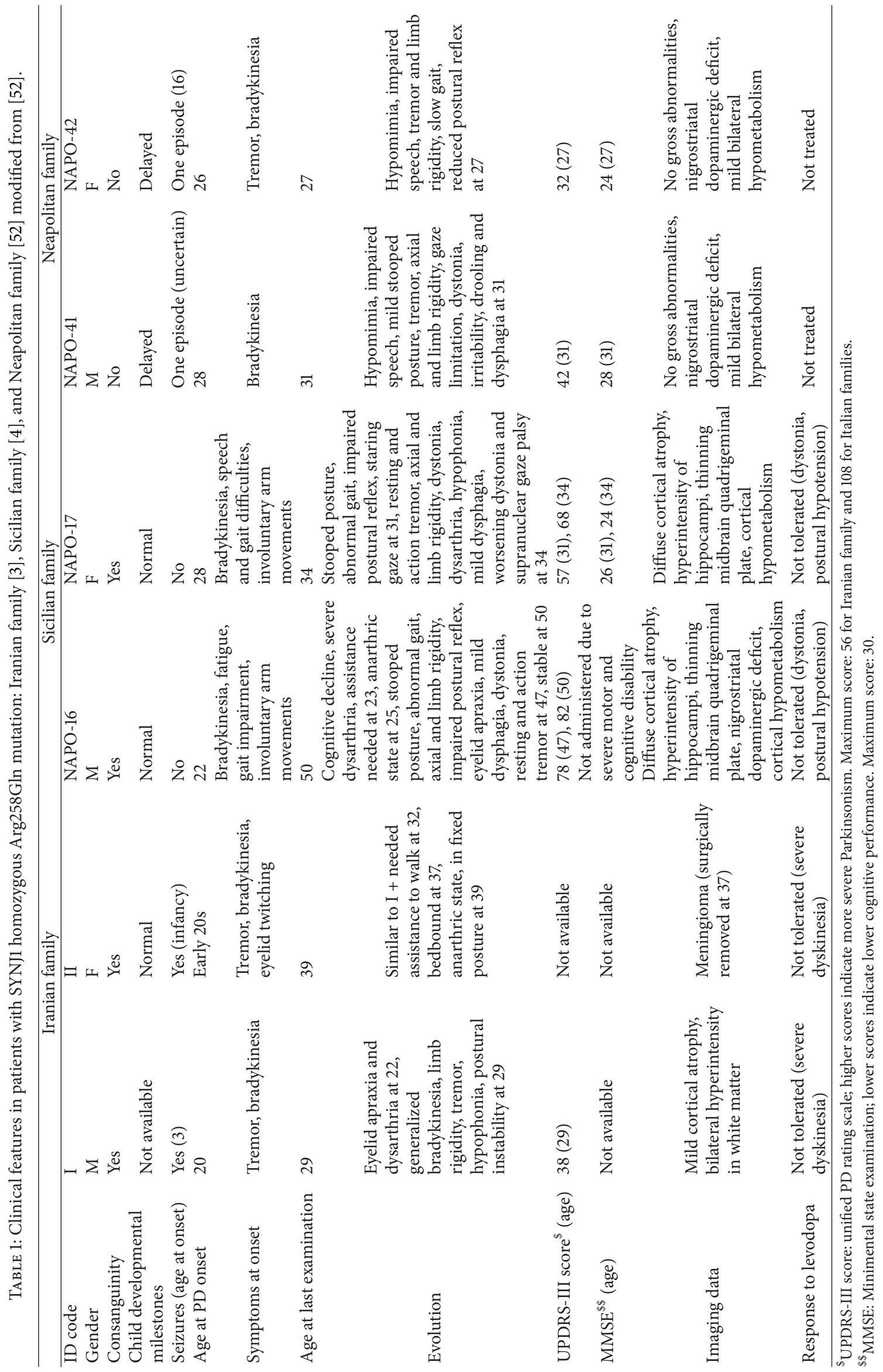




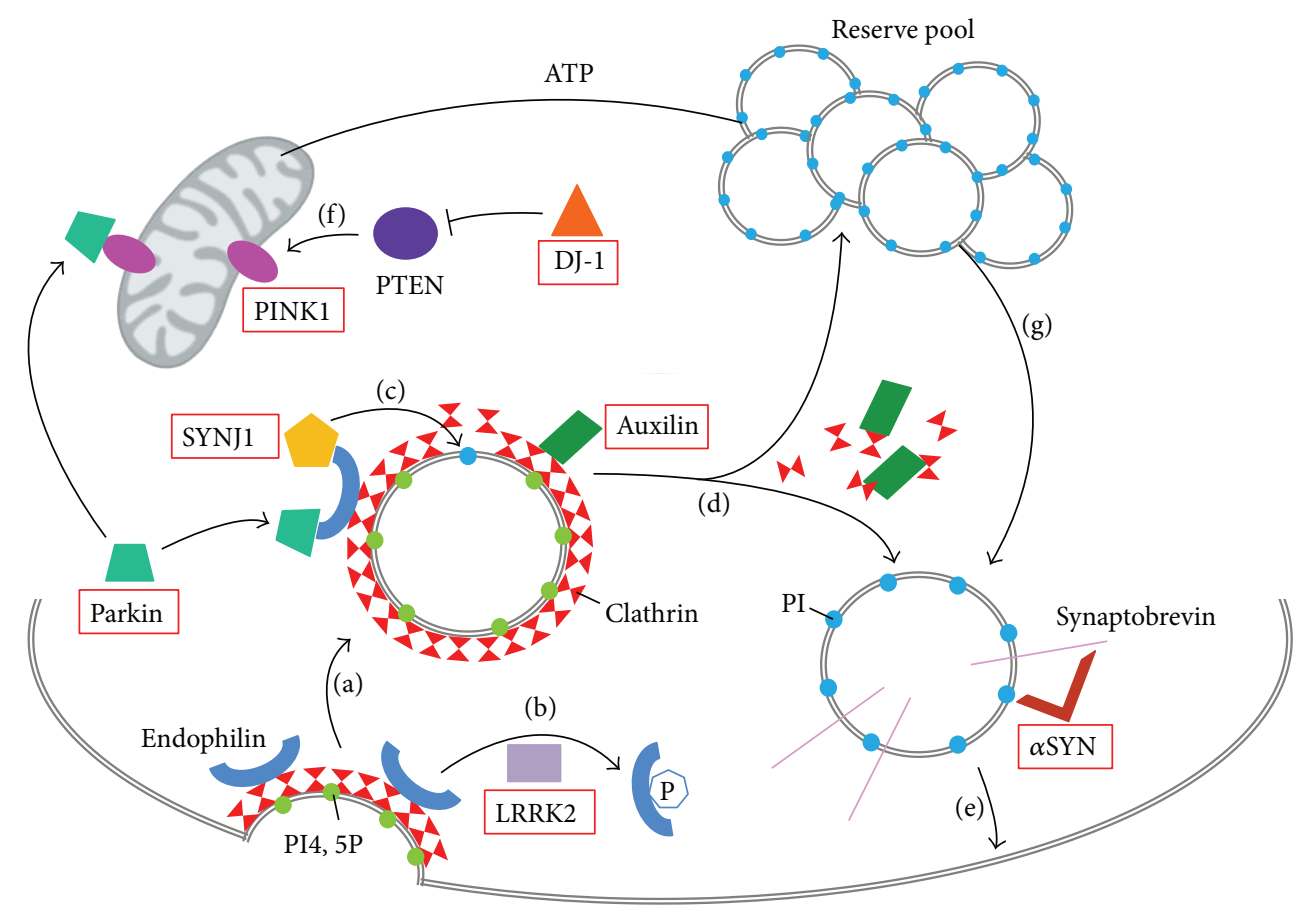

FIGURE 2: Synaptic vesicle recycling and PD genes. Schematic representation of a presynaptic terminal showing the PD genes (red boxes) and their role in synaptic vesicle recycling. (a) During endocytosis, invagination of the clathrin-coated membrane requires endophilin. Endophilin harbors several SH3 domains, which can interact with SYNJ1 PRD domain and/or parkin. (b) LRRK2 phosphorylates endophilin leading to dissociation of the later from clathrin-coated vesicles. (c) Once recruited to the coated vesicles through endophilin, SYNJ1 dephosphorylates PI4,5P into PI, shedding clathrin and its adaptor from the bilayer. (d) Uncoating of the vesicles also requires auxilin intervention and subsequent chaperoning of clathrin molecules. Then, the postendocytic vesicles can return to the reserve pool, where they undergo clustering, or return directly to the release site and enter in an exocytosis step. (e) Synaptic vesicles are docked and then fused to the membrane by means of a multiprotein complex including synaptobrevin and $\alpha$ SYN. (f) PTEN is a lipid phosphatase, which is inhibited by DJ-1, and can increase levels of the mitochondrial PINK1 protein. This pathway is involved in NMDA receptor signaling. (g) Proper mitochondrial functioning leads to ATP synthesis, necessary to mobilize the reserve pool of vesicles during synapse stimulation. PI4,5P: phosphatidylinositol 4,5bisphosphates; PI: phosphatidylinositol; ATP: adenosine triphosphate; SYNJ1: synaptojanin 1; LRRK2: leucine-rich repeat serine/threonineprotein kinase 2; PTEN: phosphatase and tensin homologue; PINK1: PTEN induced putative kinase 1; DJ-1: Parkinson's disease protein 7; $\alpha \mathrm{SYN}$ : alpha-synuclein.

in autosomal dominant $\mathrm{PD}$, as well as those responsible for autosomal recessive forms of Parkinsonism, have been implicated, directly or indirectly, in synaptic vesicle turnover (Figure 2).

Parkin, an ubiquitin ligase mutated in the most common form of early-onset autosomal recessive $\mathrm{PD}$, interacts with endophilin, which is a major binding partner of SYNJ1 (Figure 2(a)). Parkin participates in the ubiquitination of proteins present in synaptic endophilin complexes [54]. Leucinerich repeat serine/threonine-protein kinase 2 (LRRK2), which is mutated in the most common form of autosomal dominant PD, regulates endophilin association to clathrincoated vesicles through phosphorylation [55] (Figure 2(b)). Auxilin-1, which was recently identified in atypical earlyonset PD, is also a direct partner of SYNJ1 during the process of uncoating synaptic vesicles [56]. SYNJ1 and auxilin1 mutated patients show common features of early-onset Parkinsonism and seizures with other atypical symptoms. Furthermore, KO mice for each one of these genes show nearly identical phenotypes of defective synaptic vesicle recycling and severe neurological phenotype [11, 57]. Nevertheless, their roles are different in the mechanism: auxilin-1 is involved in clathrin disassembly and chaperoning, whereas SYNJ1 takes part in the adaptor shedding from the bilayer [57] (Figures 2(c) and 2(d)). Moreover, alpha-synuclein $(\alpha \mathrm{SYN})$, a presynaptic protein found accumulated in Lewy bodies in typical late-onset $\mathrm{PD}$, is also implicated in synaptic vesicle exocytosis and recycling $[58,59]$. It has been shown that $\alpha \mathrm{SYN}$ binds to phospholipids via its $\mathrm{N}$-terminus and to synaptobrevin-2 via its C-terminal extremity on synaptic vesicles surface, to promote vesicle fusion [58] (Figure 2(e)). Lastly, PINK1 (PTEN-phosphatase and tensin homologueinduced kinase 1), whose mutation is responsible for typical early-onset autosomal recessive $\mathrm{PD}$, is mostly described as a mitochondrial protein. However, it has also been shown that PINK1 deficiency affects synaptic function, as the reserve pool of synaptic vesicles is not mobilized during rapid stimulation in PINK1-deficient Drosophila [60]. Furthermore, the 
fact that PTEN (1) is a lipid phosphatase, like SYNJ1 [10, 35], (2) induces PINK1 activity, and (3) is inhibited by DJ-1 [61], another autosomal recessive associated PD protein, strongly suggests involvement of lipid metabolism in PD (Figure 2(f)).

This network of proteins associated with synaptic vesicle pathways and PD strongly supports that impaired synaptic activity, resulting from altered lipid metabolism, is a key mechanism underlining the pathology. More studies in this direction should be conducted.

Also, other proteins, which are involved in synaptic activity and interact with known PD proteins, should be considered as good candidate for PD. However, each new gene discovered as causative in PD is only incriminated in a decreasing number of families. Whole exome sequencing technology should help us find additional patients carrying these mutations, but it is most likely that we are heading towards the discovery of private PD genes, for example, one gene $=$ one family. It is going to become harder and harder to find common mutated genes in PD and therefore the validation of such candidate genes will be difficult.

\section{Conclusions}

SYNJ1 is a phosphoinositide phosphatase protein, which is required for proper synaptic activity. After being investigated as a candidate gene in bipolar disorder, Down's syndrome, and Alzheimer's disease with varying success, SYNJ1 was identified as the causative gene in three families with earlyonset atypical Parkinsonism. One single homozygous mutation has been reported so far. SYNJ1 and most of other PD proteins play a role in vesicle recycling and lipid metabolism at the synapse; thus the study of these pathways is of particular interest to dissect the neuropathological processes involved and to find potential therapeutic targets to counteract PD.

\section{Conflict of Interests}

The authors declare that there is no conflict of interests regarding the publication of this paper.

\section{Acknowledgments}

The authors thank Alexandra Kelly for critical reading of the paper. This work was supported by the French Program "Investissements d'Avenir" ANR-10-IAIHU-06.

\section{References}

[1] P. S. Mcpherson, A. J. Czernik, T. J. Chilcote et al., "Interaction of Grb2 via its Src homology 3 domains with synaptic proteins including synapsin I," Proceedings of the National Academy of Sciences of the United States of America, vol. 91, no. 14, pp. 64866490, 1994.

[2] P. S. McPherson, K. Takei, S. L. Schmid, and P. de Camilli, "p145, a major Grb2-binding protein in brain, is co-localized with dynamin in nerve terminals where it undergoes activitydependent dephosphorylation," The Journal of Biological Chemistry, vol. 269, no. 48, pp. 30132-30139, 1994.
[3] C. E. Krebs, S. Karkheiran, J. C. Powell et al., "The sacl domain of SYNJ1 identified mutated in a family with early-onset progressive parkinsonism with generalized seizures," Human Mutation, vol. 34, no. 9, pp. 1200-1207, 2013.

[4] M. Quadri, M. Fang, M. Picillo et al., "Mutation in the SYNJ1 gene associated with autosomal recessive, early-onset parkinsonism," Human Mutation, vol. 34, no. 9, pp. 1208-1215, 2013.

[5] O. Cremona, M. Nimmakayalu, C. Haffner, P. Bray-Ward, D. C. Ward, and P. P. de Camilli, "Assignment ${ }^{1}$ of SYNJ1 to human chromosome 21q22.2 and Synj12 to the murine homologous region on chromosome $16 \mathrm{C} 3-4$ by in situ hybridization," Cytogenetics and Cell Genetics, vol. 88, no. 1-2, pp. 89-90, 2000.

[6] R. M. Perera, R. Zoncu, L. Lucast, P. de Camilli, and D. Toomre, "Two synaptojanin 1 isoforms are recruited to clathrin-coated pits at different stages," Proceedings of the National Academy of Sciences of the United States of America, vol. 103, no. 51, pp. 19332-19337, 2006.

[7] A. R. Ramjaun and P. S. McPherson, “Tissue-specific alternative splicing generates two synaptojanin isoforms with differential membrane binding properties," Journal of Biological Chemistry, vol. 271, no. 40, pp. 24856-24861, 1996.

[8] P. S. McPherson, E. P. Garcia, V. I. Slepnev et al., "A presynaptic inositol-5' -phosphatase," Nature, vol. 379, no. 6563, pp. 353-357, 1996.

[9] C. Haffner, K. Takei, H. Chen et al., "Synaptojanin 1: Localization on coated endocytic intermediates in nerve terminals and interaction of its $170 \mathrm{kDa}$ isoform with Eps15," FEBS Letters, vol. 419, no. 2-3, pp. 175-180, 1997.

[10] G. di Paolo and P. de Camilli, "Phosphoinositides in cell regulation and membrane dynamics," Nature, vol. 443, no. 7112, pp. 651-657, 2006.

[11] O. Cremona, G. Di Paolo, M. R. Wenk et al., "Essential role of phosphoinositide metabolism in synaptic vesicle recycling," Cell, vol. 99, no. 2, pp. 179-188, 1999.

[12] S. Guo, L. E. Stolz, S. M. Lemrow, and J. D. York, "SAC1-like domains of yeast SAC1, INP52, and INP53 and of human synaptojanin encode polyphosphoinositide phosphatases," Journal of Biological Chemistry, vol. 274, no. 19, pp. 12990-12995, 1999.

[13] M. Mani, S. Y. Lee, L. Lucast et al., "The dual phosphatase activity of synaptojanin1 is required for both efficient synaptic vesicle endocytosis and reavailability at nerve terminals," Neuron, vol. 56, no. 6, pp. 1004-1018, 2007.

[14] B. J. Mayer and M. J. Eck, "Domains: minding your p's and q's," Current Biology, vol. 5, pp. 364-367, 1995.

[15] E. De Heuvel, A. W. Bell, A. R. Ramjaun, K. Wong, W. S. Sossin, and P. S. McPherson, "Identification of the major synaptojaninbinding proteins in brain," Journal of Biological Chemistry, vol. 272, no. 13, pp. 8710-8716, 1997.

[16] N. Ringstad, Y. Nemoto, and P. de Camilli, "The SH3p4/ Sh3p8/SH3p13 protein family: binding partners for synaptojanin and dynamin via a Grb2-like Src homology 3 domain," Proceedings of the National Academy of Sciences of the United States of America, vol. 94, no. 16, pp. 8569-8574, 1997.

[17] K. D. Micheva, A. R. Ramjaun, B. K. Kay, and P. S. McPherson, "SH3 domain-dependent interactions of endophilin with amphiphysin," FEBS Letters, vol. 414, no. 2, pp. 308-312, 1997.

[18] G. Cestra, L. Castagnoli, L. Dente et al., "The SH3 domains of endophilin and amphiphysin bind to the proline-rich region of synaptojanin 1 at distinct sites that display an unconventional binding specificity," Journal of Biological Chemistry, vol. 274, no. 45, pp. 32001-32007, 1999. 
[19] V. I. Slepnev and P. De Camilli, "Accessory factors in clathrindependent synaptic vesicle endocytosis," Nature Reviews Neuroscience, vol. 1, no. 3, pp. 161-172, 2000.

[20] T. Itoh, K. S. Erdmann, A. Roux, B. Habermann, H. Werner, and P. de Camilli, "Dynamin and the actin cytoskeleton cooperatively regulate plasma membrane invagination by BAR and F-BAR proteins," Developmental Cell, vol. 9, no. 6, pp. 791-804, 2005.

[21] I. Milosevic, S. Giovedi, X. Lou et al., "Recruitment of endophilin to clathrin-coated pit necks is required for efficient vesicle uncoating after fission," Neuron, vol. 72, no. 4, pp. 587601, 2011.

[22] G. J. K. Praefcke, M. G. J. Ford, E. M. Schmid et al., "Evolving nature of the AP2 $\alpha$-appendage hub during clathrin-coated vesicle endocytosis," EMBO Journal, vol. 23, no. 22, pp. 43714383, 2004.

[23] M. Krauß and V. Haucke, "Phosphoinositide-metabolizing enzymes at the interface between membrane traffic and cell signalling," EMBO Reports, vol. 8, no. 3, pp. 241-246, 2007.

[24] L. C. Holzhausen, A. A. Lewis, K. K. Cheong, and S. E. Brockerhoff, "Differential role for synaptojanin 1 in rod and cone photoreceptors," Journal of Comparative Neurology, vol. 517, no. 5, pp. 633-644, 2009.

[25] H. A. van Epps, M. Hayashi, L. Lucast et al., "The zebrafish nrc mutant reveals a role for the polyphosphoinositide phosphatase synaptojanin 1 in cone photoreceptor ribbon anchoring," Journal of Neuroscience, vol. 24, no. 40, pp. 8641-8650, 2004.

[26] A. A. George, S. Hayden, L. C. Holzhausen, E. Y. Ma, S. C. Suzuki, and S. E. Brockerhoff, "Synaptojanin 1 is required for endolysosomal trafficking of synaptic proteins in cone photoreceptor inner segments," PLOS ONE, vol. 9, no. 1, Article ID e84394, 2014.

[27] S. Jia, A. Muto, W. Orisme et al., "Zebrafish cacnalfa is required for cone photoreceptor function and synaptic ribbon formation," Human Molecular Genetics, vol. 23, no. 11, pp. 29812994, 2014.

[28] J. G. Trapani, N. Obholzer, W. Mo, S. E. Brockerhoff, and T. Nicolson, "Synaptojanin1 is required for temporal fidelity of synaptic transmission in hair cells," PLoS Genetics, vol. 5, no. 5, Article ID e1000480, 2009.

[29] K. Soda, D. M. Balkin, S. M. Ferguson et al., "Role of dynamin, synaptojanin, and endophilin in podocyte foot processes," Journal of Clinical Investigation, vol. 122, no. 12, pp. 4401-4411, 2012.

[30] Y. Sun, I. Tawara, M. Zhao et al., "Allogeneic T cell responses are regulated by a specific miRNA-mRNA network," Journal of Clinical Investigation, vol. 123, no. 11, pp. 4739-4754, 2013.

[31] T. W. Harris, E. Hartwieg, H. R. Horvitz, and E. M. Jorgensen, "Mutations in synaptojanin disrupt synaptic vesicle recycling," Journal of Cell Biology, vol. 150, no. 3, pp. 589-599, 2000.

[32] H. Gad, N. Ringstad, P. Löw et al., "Fission and uncoating of synaptic clathrin-coated vesicles are perturbed by disruption of interactions with the SH3 domain of endophilin," Neuron, vol. 27, no. 2, pp. 301-312, 2000.

[33] C. J. Stefan, A. Audhya, and S. D. Emr, “The yeast synaptojaninlike proteins control the cellular distribution of phosphatidylinositol (4,5)-bisphosphate," Molecular Biology of the Cell, vol. 13, no. 2, pp. 542-557, 2002.

[34] W. T. Kim, S. Chang, L. Daniell, O. Cremona, G. di Paolo, and P. de Camilli, "Delayed reentry of recycling vesicles into the fusion-competent synaptic vesicle pool in synaptojanin 1 knockout mice," Proceedings of the National Academy of Sciences of the United States of America, vol. 99, no. 26, pp. 17143-17148, 2002.

[35] P. G. Billcliff and M. Lowe, "Inositol lipid phosphatases in membrane trafficking and human disease," Biochemical Journal, vol. 461, no. 2, pp. 159-175, 2014.

[36] L. W. Gong and P. de Camilli, "Regulation of postsynaptic AMPA responses by synaptojanin," Proceedings of the National Academy of Sciences of the United States of America, vol. 105, no. 45, pp. 17561-17566, 2008.

[37] G. Matthews and P. Fuchs, "The diverse roles of ribbon synapses in sensory neurotransmission," Nature Reviews Neuroscience, vol. 11, no. 12, pp. 812-822, 2010.

[38] D. Patterson and A. C. S. Costa, "Down syndrome and genetics-a case of linked histories," Nature Reviews Genetics, vol. 6, pp. 137-147, 2005.

[39] S. V. Voronov, S. G. Frere, S. Giovedi et al., "Synaptojanin 1linked phosphoinositide dyshomeostasis and cognitive deficits in mouse models of Down's syndrome," Proceedings of the National Academy of Sciences of the United States of America, vol. 105, no. 27, pp. 9415-9420, 2008.

[40] K. T. Chang and K.-T. Min, "Upregulation of three Drosophila homologs of human chromosome 21 genes alters synaptic function: implications for Down syndrome," Proceedings of the National Academy of Sciences of the United States of America, vol. 106, no. 40, pp. 17117-17122, 2009.

[41] J.-C. Cossec, J. Lavaur, D. E. Berman et al., "Trisomy for synaptojanin 1 in down syndrome is functionally linked to the enlargement of early endosomes," Human Molecular Genetics, vol. 21, no. 14, Article ID dds142, pp. 3156-3172, 2012.

[42] S. B. Martin, A. L. S. Dowling, J. Lianekhammy et al., "Synaptophysin and synaptojanin-1 in down syndrome are differentially affected by Alzheimer's disease," Journal of Alzheimer's Disease, 2014.

[43] D. E. Berman, C. Dall'Armi, S. V. Voronov et al., "Oligomeric amyloid- $\beta$ peptide disrupts phosphatidylinositol-4,5-bisphosphate metabolism," Nature Neuroscience, vol. 11, no. 5, pp. 547$554,2008$.

[44] W.-Q. Zhao, F. Santini, R. Breese et al., "Inhibition of calcineurin-mediated endocytosis and $\alpha$-amino-3-hydroxy- 5methyl-4-isoxazolepropionic acid (AMPA) receptors prevents amyloid $\beta$ oligomer-induced synaptic disruption," Journal of Biological Chemistry, vol. 285, no. 10, pp. 7619-7632, 2010.

[45] N. Landman, S. Y. Jeong, S. Y. Shin et al., "Presenilin mutations linked to familial Alzheimer's disease cause an imbalance in phosphatidylinositol 4,5-bisphosphate metabolism," Proceedings of the National Academy of Sciences of the United States of America, vol. 103, no. 51, pp. 19524-19529, 2006.

[46] L. B. J. McIntire, D. E. Berman, J. Myaeng et al., "Reduction of synaptojanin 1 ameliorates synaptic and behavioral impairments in a mouse model of Alzheimer's disease," Journal of Neuroscience, vol. 32, no. 44, pp. 15271-15276, 2012.

[47] L. Zhu, M. Zhong, J. Zhao et al., "Reduction of synaptojanin 1 accelerates $\mathrm{A} \beta$ clearance and attenuates cognitive deterioration in an alzheimer mouse model," The Journal of Biological Chemistry, vol. 288, no. 44, pp. 32050-32063, 2013.

[48] T. Saito, F. Guan, D. F. Papolos, S. Lau, M. Klein, and C. S. J. Fann, "Mutation analysis of SYNJ1: a possible candidate gene for chromosome 21q22-linked bipolar disorder," Molecular Psychiatry, vol. 6, no. 4, pp. 387-395, 2001.

[49] P. Stopkova, J. Vevera, I. Paclt, I. Zukov, and H. M. Lachman, "Analysis of SYNJ1, a candidate gene for 21q22 linked bipolar 
disorder: a replication study," Psychiatry Research, vol. 127, no. 1-2, pp. 157-161, 2004.

[50] S. Winkler, E.-J. Vollstedt, M. Kasten, D. Alvarez-Fischer, C. Klein, and K. Lohmann, "The recurrent mutation Arg258Gln in SYNJ1 (PARK20) is not a common cause of Parkinson's disease," Journal of Neurology, vol. 261, no. 4, pp. 833-834, 2014.

[51] M. Kasten, J. Hagenah, J. Graf et al., "Cohort profile: a population-based cohort to study non-motor symptoms in Parkinsonism (EPIPARK)," International Journal of Epidemiology, vol. 42, no. 1, Article ID dys202, p. 128, 2013.

[52] S. Olgiati, A. de Rosa, M. Quadri et al., "PARK20 caused by SYNJ1 homozygous Arg258Gln mutation in a new Italian family," neurogenetics, vol. 15, no. 3, pp. 183-188, 2014.

[53] M. Picillo, A. Ranieri, G. Orefice, V. Bonifati, and P. Barone, "Clinical progression of SYNJ1-related early onset atypical parkinsonism: 3-year follow up of the original Italian family," Journal of Neurology, vol. 261, no. 4, pp. 823-824, 2014.

[54] J.-F. Trempe, C. X.-Q. Chen, K. Grenier et al., "SH3 domains from a subset of BAR proteins define a Ubl-binding domain and implicate parkin in synaptic ubiquitination," Molecular Cell, vol. 36, no. 6, pp. 1034-1047, 2009.

[55] S. Matta, K. Van Kolen, R. da Cunha et al., "LRRK2 controls an EndoA phosphorylation cycle in synaptic endocytosis," Neuron, vol. 75, no. 6, pp. 1008-1021, 2012.

[56] S. Edvardson, Y. Cinnamon, A. Ta-Shma et al., "A deleterious mutation in DNAJC6 encoding the neuronal-specific clathrinuncoating Co-chaperone auxilin, is associated with juvenile parkinsonism," PLoS ONE, vol. 7, no. 5, Article ID e36458, 2012.

[57] Y.-I. Yim, T. Sun, L.-G. Wu et al., "Endocytosis and clathrinuncoating defects at synapses of auxilin knockout mice," Proceedings of the National Academy of Sciences of the United States of America, vol. 107, no. 9, pp. 4412-4417, 2010.

[58] J. Burré, M. Sharma, T. Tsetsenis, V. Buchman, M. R. Etherton, and T. C. Südhof, " $\alpha$-Synuclein promotes SNARE-complex assembly in vivo and in vitro," Science, vol. 329, no. 5999, pp. 1663-1667, 2010.

[59] V. M. Nemani, W. Lu, V. Berge et al., "Increased expression of $\alpha$-synuclein reduces neurotransmitter release by inhibiting synaptic vesicle reclustering after endocytosis," Neuron, vol. 65, no. 1, pp. 66-79, 2010.

[60] V. A. Morais, P. Verstreken, A. Roethig et al., "Parkinson's disease mutations in PINK1 result in decreased Complex I activity and deficient synaptic function," The EMBO Molecular Medicine, vol. 1, no. 2, pp. 99-111, 2009.

[61] N. Chang, L. Li, R. Hu et al., "Differential regulation of NMDA receptor function by DJ-1 and PINK1," Aging Cell, vol. 9, no. 5, pp. 837-850, 2010. 

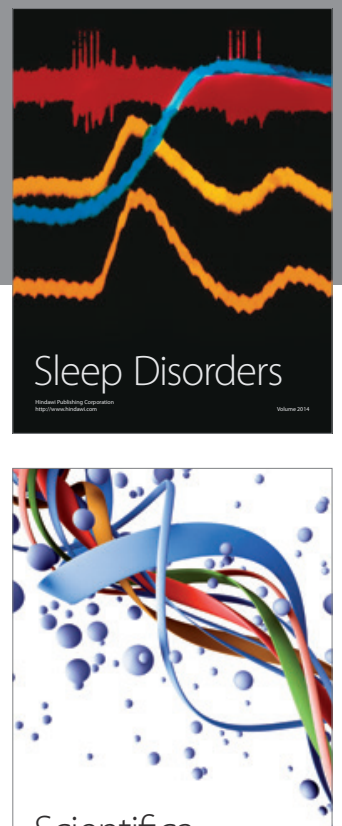

Scientifica
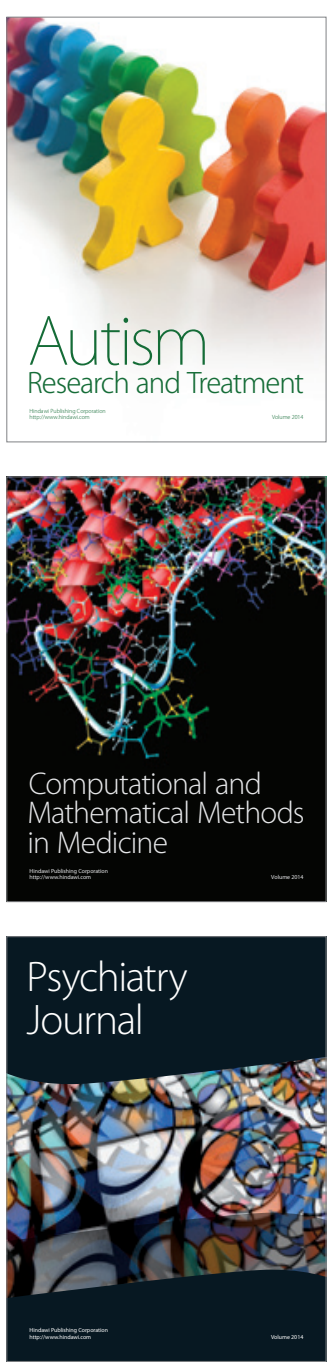
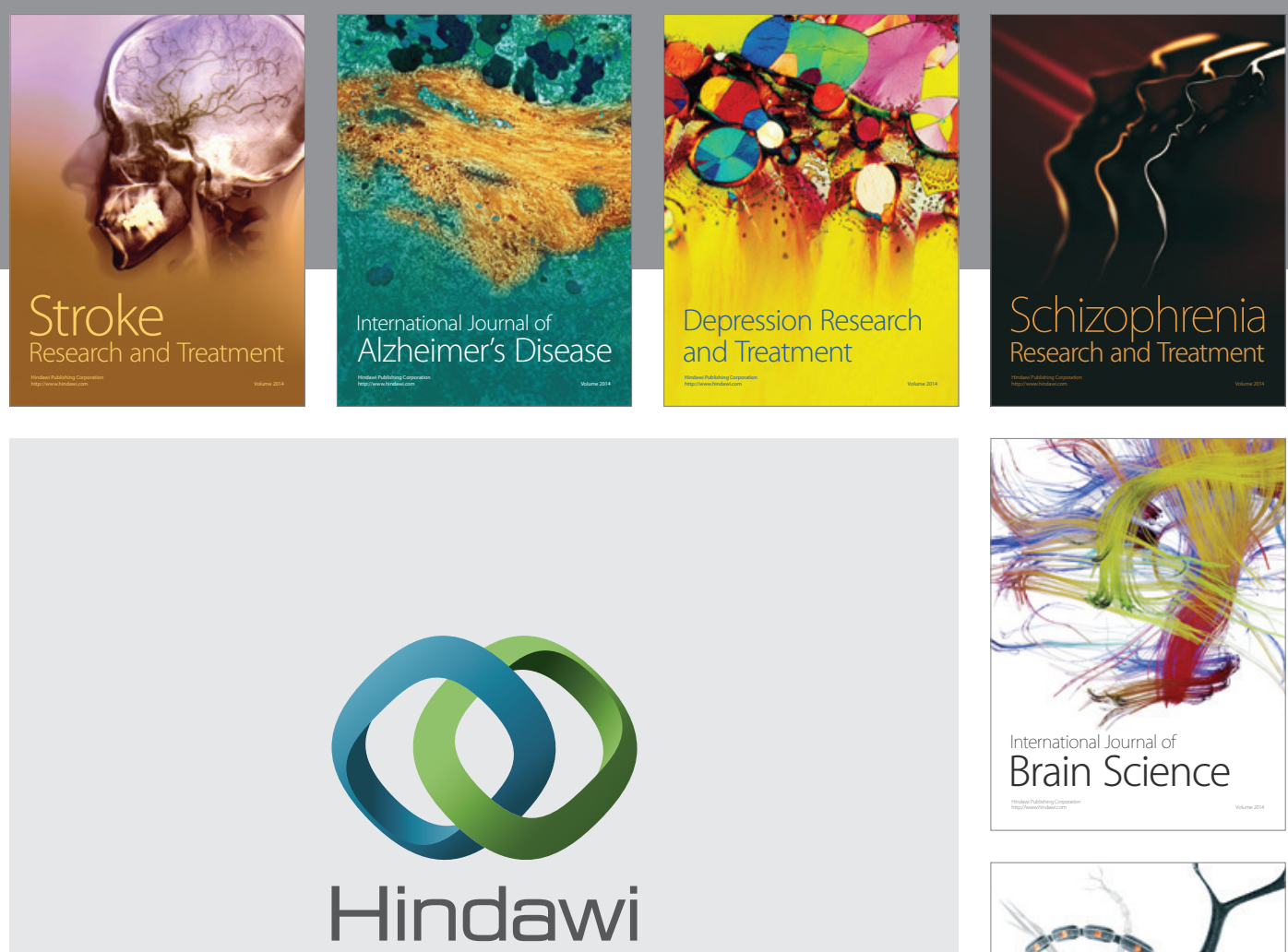

Submit your manuscripts at

http://www.hindawi.com
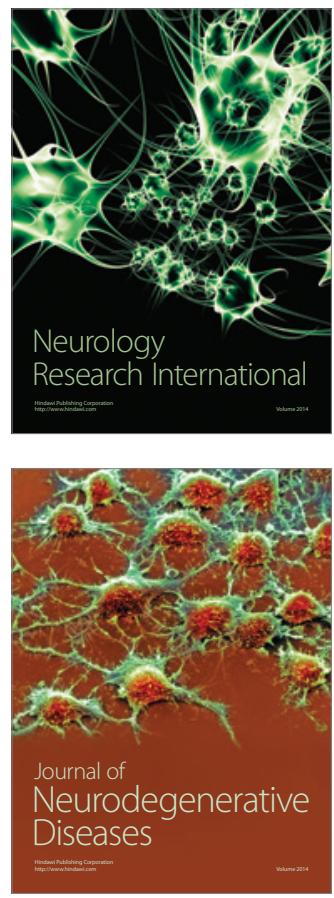

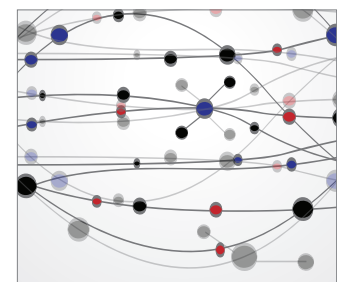

The Scientific World Journal
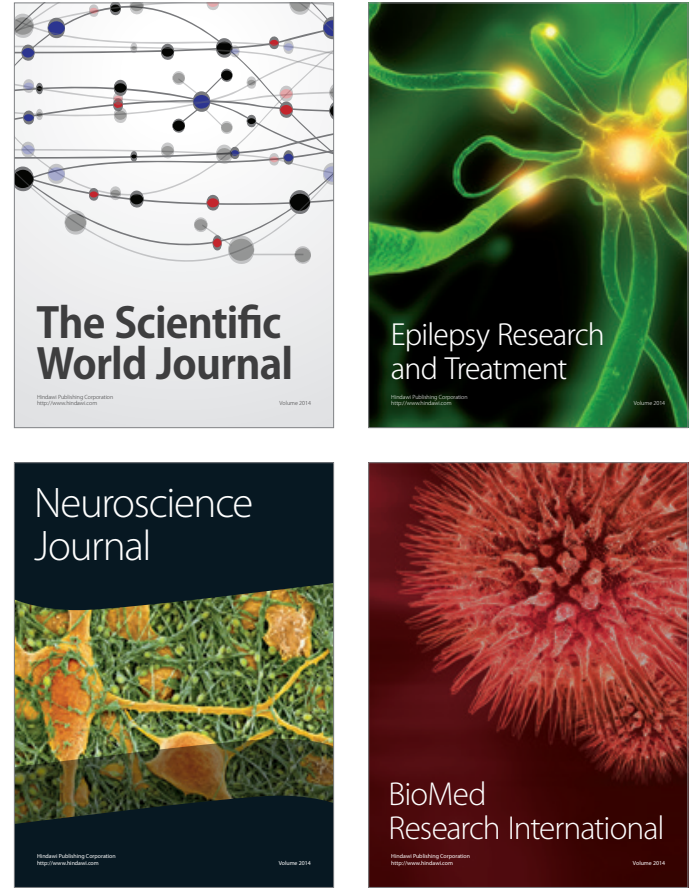

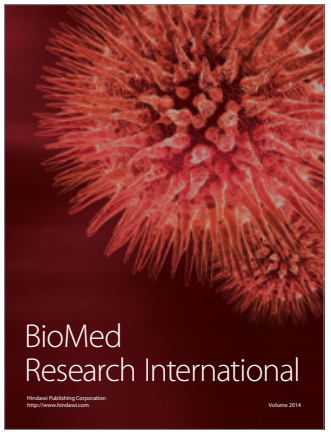

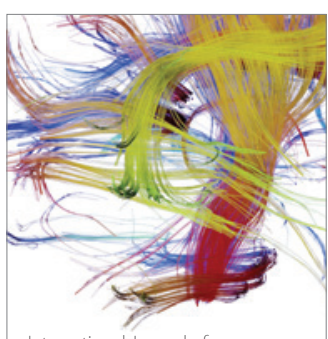

Brain Science

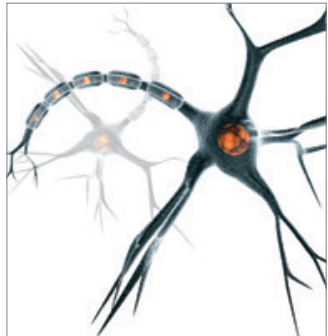

Neural Plasticity
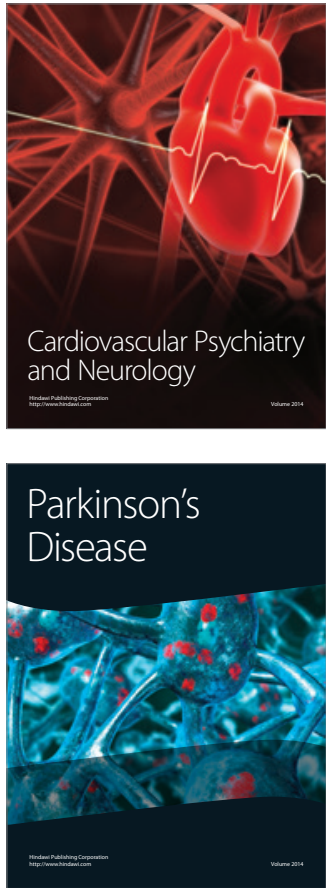